\title{
Racismo, derechos humanos, y educación superior en América Latina
}

\author{
Racism, human rights and higher \\ education in Latin America
}

Racismo, direitos humanos e ensino

superior na América Latina

DANIEL MATO

\section{Resumen}

Este artículo pone de relieve el carácter histórico estructural del racismo en las sociedades latinoamericanas y como su "naturalización" afecta las posibilidades de que personas y comunidades de pueblos indígenas y afrodescendientes puedan ejercer plenamente el derecho a la educación, con especial atención a la Educación Superior. El análisis enfatiza que este problema no solo afecta la calidad de la Educación Superior, sino también el ejercicio efectivo de derechos humanos establecidos en las normativas internacionales en la materia, y que forman parte de las constituciones nacionales y leyes de los países de la región y que los Estados tienen el deber de asegurar. Puntualiza, además que la incidencia de este problema ha sido reconocida por la 3ra. Conferencia Regional de Educación Superior de América Latina y el Caribe (CRES 2018), cuya declaración final incluyó recomendaciones respecto del papel que deberían jugar las instituciones de educación superior en la erradicación del racismo al interior de sus comunidades y en la sociedad en general. Con el fin de contribuir a hacer posible la puesta en práctica de estas recomendaciones propone contextualizar la idea de "racismo

\footnotetext{
a Universidad Nacional de Tres de Febrero - UNTREF y Consejo Nacional de Investigaciones Científicas y Técnicas - CONICET, Buenos Aires, Argentina. Doctor em Ciencias Sociales, e-mail: dmato@untref.edu.ar
} 
estructural" y desagregarla diferenciando entre factores estructurales propiamente dichos, otros específicos de los respectivos sistemas de Educación Superior, a los que denomina sistémicos, otros propios de cada IES en particular a los que denomina institucionales, y finalmente otros propios de personas que estudian o trabajan en estas instituciones, a los que denomina subjetivos.

Palabras clave: Educación Superior. Derechos Humanos. Docencia. Racismo. América Latina.

\begin{abstract}
This paper highlights the historical structural character of racism in Latin American societies and how its "naturalization" affects the possibilities of individuals and communities of indigenous and Afro-descendant peoples of fully exercising their right to education, with special attention to higher education. The analysis emphasizes that this problem does not only affect the quality of higher education, but also the effective exercise of human rights established in international regulations on the subject and which are part of the national constitutions and laws of the countries of the region and that States have the duty to ensure them. The paper also points out that the incidence of this problem was recognized by the 3rd. Regional Conference on Higher Education in Latin America and the Caribbean (CRES 2018), whose final declaration included recommendations on the role that higher education institutions should play in eradicating racism in their communities and in society in general. In order to contribute to the implementation of these recommendations, it is proposed to contextualize the idea of "structural racism" and to disaggregate it, differentiating between the structural factors themselves, some specific to the respective higher education systems, named systemic, other characteristics of each Higher Education Institute in particular, which are named institutional and, finally, some characteristics of people who study or work in these institutions, named subjective.
\end{abstract}

Keywords: Higher Education. Human Rights. Teaching. Racism. Latin America.

\title{
Resumo
}

Este artigo destaca o caráter histórico estrutural do racismo nas sociedades latinoamericanas e como sua "naturalização" afeta as possibilidades de indivíduos e comunidades de povos indígenas e afrodescendentes exercerem plenamente o direito à educação, com atenção especial ao ensino superior. A análise enfatiza que esse problema não afeta apenas a qualidade do ensino superior, mas também o exercício efetivo dos direitos humanos estabelecidos nos regulamentos internacionais sobre o assunto e que fazem parte das constituições e leis nacionais dos países da região, que os Estados têm o dever de garantir. O artigo também aponta que a incidência desse problema foi reconhecida pela $3^{a}$ Conferência Regional sobre Ensino Superior na América Latina e no Caribe (CRES 2018), cuja declaração final incluiu recomendações 
sobre o papel que as instituições de ensino superior devem desempenhar na erradicação do racismo em suas comunidades e na sociedade em geral. Para contribuir na viabilização da implementação dessas recomendações, propõe-se contextualizar a ideia de "racismo estrutural" e desagregá-la, diferenciando entre os próprios fatores estruturais, outros específicos para os respectivos sistemas de ensino superior, chamado de sistêmicos, outros característicos de cada IES em particular, que se chama de institucional e, finalmente, outras características de pessoas que estudam ou trabalham nessas instituições, a quem se chama de subjetivas.

Palavras-chave: Educação Superior. Direitos Humanos. Ensino. Racismo. América Latina.

\section{Introducción}

Desde hace siglos, en América Latina, la discriminación racial y otras modalidades de racismo afectan especialmente a personas y comunidades de pueblos indígenas y afrodescendientes, y condicionan las condiciones de inequidad y desigualdad en que deben hacer sus vidas (BELLO; RANGEL, 2000; CEPAL, 2016; HOPENHAYN, BELLO y MIRANDA, 2007; REDING, 2007).

El racismo es una ideología según la cual los seres humanos seríamos clasificables en "razas", y algunas de ellas serían "superiores" a otras. En América Latina la vigencia de esta ideología proviene del período colonial, pero no solo caracterizó a ese tiempo histórico, sino que incidió fuertemente en los sistemas jurídicos, económicos, políticos, y sociales, y la construcción de las “identidades nacionales" de las repúblicas poscoloniales. En estos procesos históricos, los sistemas e instituciones educativas de todos los niveles de las nuevas naciones jugaron, y continúan jugando, papeles clave en la difusión y reproducción de esta ideología. Las políticas, sistemas, e instituciones, de Educación Superior no escapan a estos problemas, que históricamente han contribuido a construir, y que tienen el deber y la posibilidad de contribuir a erradicar.

Los medios de comunicación masiva han hecho y continúan haciendo otro tanto. No solo suelen propagar estereotipos racistas, sino que, además, cuando informan sobre hechos de discriminación $y / o$ violencia racial lo hacen especialmente con referencia a los que acontecen en otras latitudes. Si bien hay excepciones, es poco frecuente que se ocupen de las numerosas situaciones y 
procesos racistas que afectan cotidianamente a personas y comunidades de pueblos indígenas y afrodescendientes en sus propios países. Estas situaciones y procesos no suelen ser vistos como "noticia"; porque tienen siglos ocurriendo, de manera análoga a como hasta recientemente sucedía con la discriminación y violencia contra las mujeres.

Resulta especialmente preocupante que sistemáticamente se violen los derechos humanos de estos pueblos y que esto no perturbe demasiado a otros grupos de población, o —al menos- que muy pocos de ellos no se manifiesten al respecto. También es muy preocupante, que los gobiernos de la región, como mucho, hayan creado organismos especializados donde radicar denuncias, pero no acometan campañas sistemáticas para acabar con este flagelo.

Igualmente preocupante resulta que, en pleno siglo XXI, los sistemas de educación superior aún no hayan puesto en práctica políticas efectivas - y debidamente financiadas - para acabar asegurar a estos pueblos el disfrute de los derechos educativos que forman parte de los derechos humanos establecidos en instrumentos jurídicos internacionales y constituciones nacionales. También lo es que la inmensa mayoría de las universidades y otros tipos de IES no sostengan políticas y programas deliberadamente dirigidos a lograrlo. Aparentemente, aún no se ha alcanzado suficiente conciencia acerca de hasta qué punto estas deficiencias afectan la calidad de la formación profesional y ciudadana que estas instituciones ofrecen, y de la investigación que realizan.

Tampoco parece haberse tomado conciencia de la responsabilidad de las IES en la pasividad, complicidad, y en no pocos casos protagonismo, que caracteriza las prácticas de muchos de sus egresados; por ejemplo: docentes y profesionales de la salud que dispensan tratos discriminatorios a comunidades y personas de esos pueblos; ingenieros, economistas, y otros profesionales, que dirigen empresas cuyas actividades afectan los derechos territoriales o contaminan los territorios de esos pueblos; abogados que defienden los intereses de terratenientes y corporaciones que violan los derechos de esos pueblos; y un sinfín de egresados que en tanto dirigentes sociales y políticos, y/o responsabilidades en los diversos niveles de la administración estatal de estos países ignoran y/o violan abiertamente los derechos establecidos en instrumentos jurídicos internacionales que han sido ratificados por 
los respectivos Estados, los cuales, según los casos, tienen rango constitucional o de ley orgánica en todos los países latinoamericanos ¿Cómo se explica que ocurra todo esto y la mayoría de las universidades y otras IES aún no hayan tomado medidas al respecto? ¿Cómo se explica que estos problemas no ocupen un lugar destacado en los debates y agendas institucionales?

\section{La naturalización del racismo en las sociedades latinoamericanas}

En las sociedades latinoamericanas se ha "naturalizado" que las personas y comunidades de estos pueblos no solo deban enfrentar comentarios y miradas denigrantes, o prejuicios que afectan sus posibilidades de acceder en pie de igualdad a oportunidades laborales, servicios educativos, de salud y justicia. También se ha "naturalizado" que hayan sido desplazados de sus territorios originales y hoy vivan en otros en condiciones ambientales y sanitarias deplorables. Así mismo, se ha "naturalizado" que sus tierras sean usurpadas por terratenientes y empresas forestales, para lo cual usualmente emplean medios violentos e incluso llegan a asesinarlos. De manera semejante parece haberse "naturalizado" que su hábitat sea contaminado por las actividades de corporaciones mineras y petroleras, o que sus niñas sean víctimas de violaciones, o que sus miembros trabajen en la agricultura sin que se respeten sus derechos laborales, o que las escuelas y hospitales cercanas a sus comunidades presenten condiciones incluso peores que las que presentan los que atienden a otros sectores sociales nada favorecidos por las políticas y presupuestos públicos. Hasta se ha naturalizado que ni siquiera haya escuelas y hospitales cercanos a sus lugares de residencia, así como que no haya universidades u otros tipos de instituciones de educación superior (IES).

Todo eso ha sido "naturalizado", y, de este modo, a pocos sorprende que las personas indígenas y afrodescendientes frecuentemente no completen su escolaridad, o que deban asistir a escuelas en las que la instrucción se ofrece en una lengua extranjera; es decir, en castellano o portugués, según el caso, cuando en sus hogares se habla una lengua indígena. Significativamente, tampoco sorprende que en esas escuelas nada se aprenda sobre la historia de sus pueblos y que las ilustraciones 
de los libros representen situaciones y personas que no permite que se sientan identificados, lo cual, desde luego se repite y refuerza en la televisión, en las vallas publicitarias instaladas al margen de las carreteras, como también en la mayoría de las imágenes que pueden ver en los medios digitales a los que crecientemente acceden. De este modo, a pocos sorprende que estas personas no solo sufran discriminación, sino que incluso vivan sintiéndose extranjeros en la porción del planeta en la cual, desde hace milenios, han habitado sus antepasados.

En el marco de estas "naturalizaciones", tampoco sorprende que la formación ofrecida por los sistemas educativos dirigidos a otros sectores sociales nada enseñe sobre las historias, conocimientos, sistemas de valores, formas de organización social, y otros elementos característicos de la historia y presente de esos pueblos. O bien que, cuando lo hace, refiera a ellos como pueblos extintos, o bárbaros, indolentes, atrasados, obstáculos al progreso, y otras representaciones descalificadoras. De este modo, el resto de la ciudadanía de los respectivos países pasa su vida desinformada sobre estos pueblos, o, peor aún, prejuiciosamente informada. Por esto, contra las evidencias censales, también ocurre que a pocos sorprende que la propia existencia contemporánea de esos pueblos sea negada, no solo en la educación básica, media, y superior, sino también en las alocuciones púbicas de numerosos dirigentes sociales, económicos y políticos.

Que dirigentes políticos y sociales y altos funcionarios de los estados vean nuestras sociedades de esa manera es desde luego responsabilidad personal de cada una de estas personas. Pero la mayoría de ellas han egresado de universidades u otros tipos de IES, las cuales evidentemente tampoco les educaron mejor. Las instituciones y políticas de educación superior no escapan a estos problemas. Ellos se expresan no solo en prejuicios y descalificaciones, sino también en la exclusión de las historias, lenguas y conocimientos de esos pueblos en los planes de estudio. También lo hacen en la escasa participación de personas indígenas y afrodescendientes entre el estudiantado y los cuerpos docentes y de autoridades y funcionarios.

¿Por qué nada de esto sorprende? Porque el racismo es un elemento constitutivo de estas sociedades, que continúa estructuralmente caracterizando su vida contemporánea. 
Si bien en algunos países los comportamientos abiertamente discriminatorios hacia estas personas y comunidades son menos habituales que décadas atrás, existen desventajas históricamente acumuladas, normas, mecanismos institucionales, prejuicios, y prácticas que, de maneras frecuentemente imperceptibles, continúan reproduciendo inequidades que afectan a estos pueblos, sus miembros y comunidades. Para hacer referencia a ese amplio y diverso conjunto de problemas se suele emplear la expresión "racismo estructural".

Pero, si bien el concepto "racismo estructural" puede ser apropiado para enfatizar la densidad histórica de esta ideología y que esta afecta las bases mismas de nuestras sociedades, puede resultar problemático para visibilizar las múltiples formas concretas en que el racismo impregna toda la vida social, así como el hecho de que no solo opera de maneras explícitas, sino también de otras menos visibles, tan naturalizadas a lo largo de los siglos, que algunas de ellas resultan inconscientes. En esto se asemeja al machismo, que frecuentemente hace ver como "naturales" circunstancias que de ningún modo lo son, sino que resultan de procesos sociales, históricamente instituidos, incluso en nuestras formas de ver la vida, el mundo social. En una sección posterior de este texto se ofrecen ejemplos de algunas de las modalidades concretas en que el racismo menoscaba las posibilidades de acceso de personas indígenas y afrodescendientes a la Educación Superior y su exitosa graduación, a la vez que afectan la calidad de la formación que las IES proveen y la investigación que realizan. Pero antes es necesario comentar brevemente acerca de los derechos educativos de esos pueblos consagrados en diversos instrumentos jurídicos internacionales.

\section{Derechos educativos de los pueblos indígenas y afrodescendientes que los Estados tienen el deber de asegurar}

En 1948 la Asamblea General de Naciones Unidas adoptó la Declaración Universal de los Derechos Humanos cuyo artículo 26 establece el derecho a la educación como uno de ellos. En la década de 1960, adoptó otros dos instrumentos jurídicos que, entre otros avances, perfeccionaron el alcance de dicho artículo. Debe 
tenerse en cuenta que esa declaración al igual que estos nuevos instrumentos han sido ratificados por todos los países latinoamericanos y son de cumplimiento obligatorio para todos ellos, al igual que otros aprobados posteriormente por esa instancia y por la Organización Internacional del Trabajo (OIT). Más aún, ellos tienen rango constitucional o de ley orgánica en todos los países signatarios.

Posteriormente se aprobaron otros instrumentos que complementan estos derechos, entre otros, por ejemplo, la Declaración de Naciones Unidas sobre los derechos de las personas pertenecientes a minorías nacionales o étnicas, religiosas y lingüísticas, la Declaración y Programa de Acción. Conferencia Mundial contra el Racismo, la Discriminación Racial, la Xenofobia y las Formas Conexas de Intolerancia, la Declaración de Naciones Unidas sobre los Derechos de los Pueblos Indígenas, la Proclamación del Decenio Internacional para los Afrodescendientes, y la Declaración Americana sobre los Derechos de los Pueblos Indígenas.

Sin embargo, el racismo que impregna los más diversos ámbitos de las sociedades latinoamericanas se expresa, entre otras formas, en la ignorancia de dirigentes políticos y funcionarios públicos respecto de los derechos de esos pueblos establecidos en dichos instrumentos y del deber de los Estados de garantizarlos.

Estudios realizados por grupos de expertos y relatores de la Organización de Naciones Unidas (ONU) llevan a concluir no solo que estos derechos están escasamente asegurados en estos países, sino que frecuentemente ocurre que existen funcionarios públicos con responsabilidades en la materia que manejan el tema como si el aseguramiento de estos derechos fuera un asunto opcional, cuyo cumplimiento dependiera de actitudes solidarias y disponibilidades presupuestarias, e incluso no faltan quienes cuestionen la pertinencia de los mismos.

Un estudio realizado en 2010 por un grupo de expertos para el Instituto Internacional de la UNESCO para la Educación Superior en América Latina y el Caribe (UNESCO-IESALC) respecto de diez países de la región (MATO, 2012), y otro realizado en 2017 por otro grupo de expertos, por encargo conjunto de ese mismo instituto y del Comité Organizador la 3ra. Conferencia Regional de Educación Superior de América Latina y el Caribe (CRES, 2018) con cobertura para todos los países continentales de la región (MATO, 2018), constataron la existencia de importantes brechas entre los derechos educativos de estos pueblos consagrados 
en las respectivas constitucionales nacionales, su expresión en leyes, la de estas en políticas públicas, y la efectiva puesta en práctica de estas últimas. Los derechos educativos de los pueblos indígenas y afrodescendientes son escasamente honrados por los respectivos Estados. Esto también ha sido consignado en informes de relatores especiales de la Organización de Naciones Unidas dedicados a dar cuenta de la situación de estos pueblos en la región (ONU, 2005, 2010, 2013, 2014a, 2019)

Para comprender apropiadamente el alcance de los mencionados derechos y su importancia para el tema que nos ocupa, resulta conveniente comentar el contenido de dichos instrumentos.

El artículo 26 de la Declaración Universal de los Derechos Humanos establece que "el acceso a los estudios superiores será igual para todos, en función de los méritos respectivos" y que uno de los objetivos principales de la educación debe ser favorecer "la comprensión, la tolerancia y la amistad entre todas las naciones y todos los grupos étnicos o religiosos" (ONU, 1948). Más recientemente, el artículo 13 del Pacto Internacional de Derechos Económicos, Sociales y Culturales estableció que los Estados "reconocen el derecho de toda persona a la educación", y convienen en que esta "debe orientarse hacia el pleno desarrollo de la personalidad humana y del sentido de su dignidad, y debe fortalecer el respeto por los derechos humanos y las libertades fundamentales" (ONU, 1966).

En contraste con los derechos así establecidos, en América Latina, las políticas, sistemas, e instituciones de educación, no garantizan a todos los grupos de población su acceso igualitario a la misma, y menos aún a la educación superior. Si bien, las normas vigentes no excluyen explícitamente a ninguno de ellos, varios grupos de población resultan excluidos en la práctica, debido a diversos tipos de factores. Entre otros, la localización de los centros educativos, por ejemplo, frecuentemente acaba excluyendo de hecho a pueblos indígenas y afrodescendientes de sus derechos educativos, y especialmente los de acceso a Educación Superior. Adicionalmente, los planes de estudio de las carreras profesionales no suelen contener contenidos o trayectos formativos orientados por el objetivo de favorecer la comprensión, tolerancia y la amistad entre los grupos étnicos de los respectivos países. Esto resulta evidente no sólo porque estos principios no forman parte de los mismos, sino que, además, esos planes de estudio no incluyen los conocimientos, 
modos de producción de conocimiento, lenguas y otros elementos propios de las respectivas culturas.

Adicionalmente, el artículo 1 de la Convención Internacional sobre la Eliminación de todas las Formas de Discriminación Racial establece que la expresión "discriminación racial" denota toda distinción basada en referentes de "raza, color, linaje u origen nacional o étnico" que tenga por objeto o resultado "anular o menoscabar el reconocimiento, goce o ejercicio, en condiciones de igualdad, de los derechos humanos y libertades fundamentales en las esferas política, económica, social, cultural o en cualquier otra esfera de la vida pública." Adicionalmente, especifica que "no se considerarán como medidas de discriminación racial" aquellas que se adopten "con el fin exclusivo de asegurar el adecuado progreso de ciertos grupos raciales o étnicos" con el propósito de "garantizarles en condiciones de igualdad, el disfrute o ejercicio de los derechos humanos y de las libertades fundamentales". Adicionalmente, el artículo 2 prevé la necesidad de que los Estados tomen medidas especiales para asegurar que "ciertos grupos raciales o de personas pertenecientes a estos grupos” con el propósito de "garantizar en condiciones de igualdad el pleno disfrute por dichas personas de los derechos humanos y de las libertades fundamentales." (ONU, 1965).

Si bien en los países latinoamericanos actualmente no existen leyes u otros instrumentos normativos que impidan el acceso de personas indígenas y afrodescendientes a Educación Superior, las políticas de Educación Superior, y los presupuestos asignados para ponerlas en práctica, tienen por resultado menoscabar sus posibilidades de ejercer los mencionados derechos en condiciones de igualdad. Porque, excepto unas cuantas iniciativas de limitado alcance en solo algunos de ellos (Brasil, Ecuador, México, Nicaragua y Perú), estas políticas no incluyen medidas apropiadas, ni se asignan presupuestos suficientes, para asegurar sus posibilidades de ejercer sus derechos educativos en condiciones de igualdad, especialmente en lo que respecta a Educación Superior.

Por otra parte, el Convenio 169 de la Organización Internacional del Trabajo (OIT) sobre Pueblos Indígenas y Tribales en Países Independientes (OIT, 1989), que tiene rango constitucional o de ley orgánica en todos los países que lo han ratificado, establece un conjunto de derechos educativos para estos pueblos que 
continúan sin ser debidamente garantizados por los estados latinoamericanos, como lo muestran los antes mencionados estudios e informes de la ONU.

Por ejemplo, el artículo 26 de este Convenio establece que a los miembros de los pueblos indígenas se les deberá garantizar "la posibilidad de adquirir una educación a todos los niveles, por lo menos en pie de igualdad con el resto de la comunidad nacional” (Itálicas añadidas, D. M.). Por otra parte, el artículo 31 estipula que "deberán adoptarse medidas de carácter educativo en todos los sectores de la comunidad nacional $[. .$.$] con objeto de eliminar los prejuicios que pudieran tener$ con respecto a esos pueblos". También determina que se deberá asegurar que los libros y demás material didáctico “ofrezcan una descripción equitativa, exacta e instructiva de las sociedades y culturas" de estos pueblos (OIT, 1989). En síntesis, este convenio dispone que los Estados deben garantizar a los pueblos indígenas acceso a educación “a todos los niveles”, y que deben educar a toda la población para eliminar prejuicios respecto de estos pueblos. Los mencionados estudios y reportes de la ONU dejan claro que esto no se cumple.

La misma situación se incumplimiento se plantea respecto de la Declaración de Naciones Unidas sobre los derechos de las personas pertenecientes a minorías nacionales o étnicas, religiosas y lingüísticas. El artículo 4 de esta declaración estipula que los Estados deberán adoptar medidas apropiadas para que "las personas pertenecientes a minorías puedan tener oportunidades adecuadas [...] de recibir instrucción en su idioma materno." Adicionalmente, determina que deberán "promover el conocimiento de la historia, las tradiciones, el idioma y la cultura de las minorías que existen en su territorio" (ONU, 1992).

Otro tanto, cabe afirmar respecto de las recomendaciones establecidas en la Proclamación del Decenio Internacional para los Afrodescendientes (2015-2024), las cuales si bien no tienen carácter vinculante para los Estados, poseen importante significación ético-política. Entre otras recomendaciones, el numeral 12 de este documento estipula que, mediante la investigación y la educación, "los Estados deberían promover un mayor conocimiento y el reconocimiento y respeto de la cultura, la historia y el patrimonio de los afrodescendientes." Adicionalmente, establece que deberían "abogar para que la historia y las contribuciones de los afrodescendientes se incluyan de forma completa y precisa en los planes de estudio"; 
así como procurar que los libros de texto y materiales educativos reflejen "los hechos históricos relativos a tragedias y atrocidades del pasado, en particular la esclavitud." (ONU, 2014b).

\section{Racismo y Educación Superior}

En contraste con esos los derechos y recomendaciones antes comentados, las situaciones de discriminación y violencia racial aún hoy resultan frecuentes en numerosas IES de América Latina.

Por ejemplo, en una entrevista realizada por el autor de este artículo en 2017, le fue referido un caso que ocurrió ese mismo año en una prestigiosa universidad brasilera. El testimonio ofrecido por una docente de esa universidad relata el caso de un estudiante "blanco" que depositó agresivamente una banana en el pupitre en el que estaba estudiando un estudiante "negro" y agresivamente le dijo: "macaco cotista"; es decir: "mono de cuota". Con esta expresión, el estudiante "blanco" hacía referencia a los programas de cupos reservados a estudiantes indígenas, negros, y otros de sectores sociales excluidos.

$\mathrm{El}$ anterior es un ejemplo de violencia racista interpersonal explícita. Pero no solo en Brasil, sino en todos los países de la región también abundan ejemplos de otras modalidades de racismo que suelen resultar menos "visibles". Son modalidades de racismo que hacen parte de los procesos constitutivos de las naciones americanas, que se construyeron a lo largo de la historia despojando a estos pueblos de sus hábitats, es decir de sus territorios y lo que en ellos hacia posible su vida. Estos procesos se expresan en que estos pueblos crecientemente hayan visto limitado su acceso a los que históricamente fueron sus medios de vida propios, y les ha forzado a insertarse en sistemas económicos ajenos, a los que se incorporan en situaciones marcadamente desventajosas.

El racismo como ideología se ha encargado de que esto no lo veamos y además garantiza su reproducción, la cual además resulta posible gracias a diseños y prácticas institucionales a través de los cuáles personas de estos pueblos tropiezan con obstáculos que no deben enfrentar otros sectores sociales. Por ejemplo, la 
carencia o insuficiencia de oportunidades de Educación Intercultural Bilingüe contextualmente apropiada y de calidad afecta las posibilidades de formación y empleo de personas que provienen de hogares en los cuales se habla su propia lengua y que se ven forzadas a educarse en una lengua extranjera (castellano o portugués, según el caso), y además a hacerlo en sistemas educativos que -como se señaló más arriba- no solo no incluyen sus conocimientos, valores y visiones de mundo, sino que además propagan visiones estereotipadas y negativas de sus pueblos.

El racismo no solo afecta las posibilidades de que personas indígenas y afrodescendientes accedan a la Educación Superior y logren graduarse en los lapsos esperables. También afecta la calidad de la formación que las universidades y otras IES ofrecen, la investigación que realizan, y su papel en la formación de ciudadanos y de opinión pública.

Resulta paradójico que en las facultades de Farmacia no se estudien los conocimientos de pueblos indígenas acerca de aplicaciones terapéuticas de especies vegetales, pero que corporaciones farmacéuticos transnacionales se ocupen de obtenerlos y patentarlos en su propio beneficio. Igualmente paradójico resulta que en las facultades de Derecho nada se aprenda sobre las formas de administración de justicia de estos pueblos, en los cuales no hay cárceles, pero esto de ningún modo significa que las infracciones a las normas sean toleradas, sino que son manejadas de otras formas, ni mejores, ni peores, otras. En vista de esta diferencia y del reconocido fracaso de las cárceles para mejorar la vida de las respectivas sociedades, cabría esperar que estas otras formas de administración de justicia al menos fueran estudiadas en las facultades de Derecho, pero no lo son. Por otro lado, el racismo impregna la formación de docentes y por su intermedio se irradia hacia todo el sistema educativo, y así también a toda la sociedad. Otro tanto ocurre con la formación de periodistas y en general de comunicadores sociales.

Estos ejemplos permiten apreciar que el racismo no solo perjudica de manera directa a las personas y comunidades de pueblos indígenas y afrodescendientes, sino además, de diversas formas, a cada sociedad en su conjunto, que se priva de sus conocimientos, lenguas y contribuciones a la solución de importantes desafíos 
ambientales y sociales. Además, la pervivencia del racismo afecta la calidad democrática y la paz en estas sociedades.

\section{La erradicación del racismo en las recomendaciones de la 3ra. Conferencia Regional de Educación Superior de América Latina y el Caribe}

En vista de los problemas señalados en secciones anteriores de este artículo, y de lo establecido en los instrumentos internacionales antes mencionados, la Declaración Final de la 3ra. Conferencia Regional de Educación Superior de América Latina y el Caribe incluyó varias recomendaciones respecto del tema que nos ocupa. Para valorar apropiadamente la importancia de esas recomendaciones resulta necesario considerar la importancia de ese evento y de la declaración emitida.

La primera de estas reuniones cumbre de los sistemas de educación superior de América Latina se realizó en La Habana, Cuba, en 1996, la segunda en Cartagena de Indias, Colombia, en 2008, y la tercera en Córdoba, Argentina en 2018. La realización de estos eventos responde a varios objetivos, favorecer el intercambio de información y las deliberaciones entre actores clave de los sistemas e IES de la región, procurar consensuar algunas orientaciones o lineamientos generales de políticas en la materia, y fijar posiciones en vista la siguiente cumbre mundial de Educación Superior. Dada la importancia de estos objetivos, el período preparatorio de estas reuniones suele extenderse por más de un año, y durante el mismo se realizan estudios preparatorios para asegurar el éxito del encuentro en cuestión.

La tercera CRES, usualmente referida como CRES 2018, fue organizada en torno a siete ejes temáticos, para cada uno de los cuales se designó un equipo de especialistas al que se le encargó realizar el respectivo estudio. Significativamente, uno de estos ejes temáticos fue dedicado a analizar el campo que el Comité Organizador de la conferencia nombró "Educación Superior, Diversidad Cultural e Interculturalidad". El equipo dedicado a analizar este campo y formular un primer cuerpo de recomendaciones al respecto estuvo constituido por trece especialistas en el tema, provenientes de doce países latinoamericanos. El estudio realizado, como 
los efectuados por los demás equipos, fue publicado de manera conjunta por el Instituto Internacional de la UNESCO para la Educación Superior en América Latina y el Caribe (UNESCO-IESALC) y la Universidad Nacional de Córdoba, institución anfitriona y organizadora local de la reunión (MATO, 2008), y sus conclusiones y recomendaciones fueron presentadas en un simposio de dos días de duración, el cual contó con la participación de más de seiscientos especialistas, autoridades de IES y organismos gubernamentales, estudiantes, docentes y representantes de organizaciones de trabajadores no docentes de las IES de la región.

De las deliberaciones de ese simposio surgió el cuerpo de recomendaciones en la materia que, integrado con los de los otros ejes, pasó a formar parte de la declaración final, la cual fue aprobada por una reunión plenaria en la que participaron más de cinco mil representantes de treinta y tres países, incluyendo ministros y secretarios de educación y educación superior, autoridades de universidades y otras IES, docentes, estudiantes, investigadores y otros especialistas en el tema, representantes de organizaciones de docentes, estudiantes y trabajadores del sector.

Fue del modo antes descripto que se aprobaron las recomendaciones en la materia, de las cuales a continuación se presenta una versión resumida

Las políticas y las instituciones de educación superior deben contribuir proactivamente a desmontar todos los mecanismos generadores de racismo, sexismo, xenofobia, y todas las formas de intolerancia y discriminación. [...]

Es necesario promover la diversidad cultural y la interculturalidad en condiciones equitativas y mutuamente respetuosas. El reto no es solo incluir en las instituciones de educación superior a mujeres, personas con discapacidad, miembros de pueblos indígenas y afrodescendientes e individuos de grupos sociales históricamente discriminados, sino transformarlas para que sean social y culturalmente pertinentes.

Estos cambios deben asegurar la incorporación en las instituciones de educación superior de las cosmovisiones, valores, conocimientos, saberes, sistemas lingüísticos, formas de aprendizaje y modos de producción de conocimiento de dichos pueblos y grupos sociales. [...]

Es ineludible reconocer y valorar las epistemologías, modos de aprendizaje y diseños institucionales propios de pueblos indígenas y afrodescendientes, comunidades campesinas y otras socioculturalmente diferenciadas. [...] 
Existe una importante deuda histórica de los Estados y sociedades latinoamericanas y caribeñas con los pueblos indígenas y afrodescendientes. Si bien sus derechos son reconocidos en numerosos instrumentos internacionales y en la mayoría de las constituciones nacionales, existe un alarmante déficit en el disfrute efectivo de los mismos, incluso en la educación superior. Las instituciones de educación superior deben garantizar el disfrute efectivo de estos derechos y educar a la población en general, y particularmente a sus comunidades, contra el racismo y todas las formas de discriminación y formas conexas de intolerancia.

Es fundamental promover y facilitar el aprendizaje de las lenguas de pueblos indígenas y afrodescendientes y su uso efectivo en las prácticas de docencia, investigación y extensión. La formación de docentes interculturales bilingües para todos los niveles de los sistemas educativos es un deber prioritario de la educación superior (CRES, 2018)

Como puede observarse, la declaración de la CRES 2018 enfatiza en la necesidad de que "las políticas y las instituciones de educación superior deben contribuir proactivamente a desmontar todos los mecanismos generadores de racismo" y en la de que las IES deben garantizar a pueblos indígenas y afrodescendientes el disfrute efectivo de sus derechos educativos, y en que deben “educar a la población en general, y particularmente a sus comunidades, contra el racismo y todas las formas de discriminación y formas conexas de intolerancia. De manera convergente y complementaria, las recomendaciones insisten en la necesidad de que esas políticas e instituciones promuevan "la diversidad cultural y la interculturalidad en condiciones equitativas y mutuamente respetuosas" y en que con este propósito las IES se transformen para ser "social y culturalmente pertinentes". A lo anterior agrega que estas transformaciones deben asegurar la incorporación en las IES “de las cosmovisiones, valores, conocimientos, saberes, sistemas lingǘsticos, formas de aprendizaje y modos de producción de conocimiento de dichos pueblos y grupos sociales". También señala que las políticas deben "reconocer y valorar las epistemologías, modos de aprendizaje y diseños institucionales propios de pueblos indígenas y afrodescendientes, comunidades campesinas y otras socioculturalmente diferenciadas". Finalmente, enfatiza en la necesidad de "promover y facilitar el aprendizaje de las lenguas de pueblos indígenas y afrodescendientes y su uso efectivo" en la Educación Superior, y en que "la formación de docentes interculturales bilingües para todos los niveles de los sistemas educativos es un deber prioritario de la educación superior". 


\section{La erradicación del racismo de la Educación Superior demanda contextualizar la idea de "racismo estructural"}

La Declaración de la CRES 2018 señala explícitamente la incidencia del racismo en la Educación Superior, el cual que convoca a erradicar, y además reconoce la vigencia de los derechos educativos de los pueblos indígenas y afrodescendientes, cuyo aseguramiento apela a lograr. En concordancia con esto, propone una serie de recomendaciones concretas orientadas a transformar las políticas y las instituciones de Educación Superior. El desafío es ponerlas en práctica.

Para llevar estas recomendaciones a la práctica resulta provechoso revisar la idea de "racismo estructural", porque esta manera de nombrar la problemática en cuestión la sitúa en una dimensión “estructural”, respecto de la cual no resulta evidente cómo intervenir.

En este sentido, en lo que hace específicamente a la Educación Superior, y sin con esto pretender establecer ningún tipo e teoría de alcance general, parece provechoso situar la idea de "racismo estructural" respecto de los contextos de actuación de los diversos actores sociales, individuales, colectivos, e institucionales con incidencia en este campo.

Resulta útil diferenciar entre modalidades de racismo de carácter propiamente estructural, de otras de carácter sistémico, es decir propias de cada sistema de Educación Superior, y de aquellas otras propias de cada IES en particular. Esta puesta en contexto puede permitir identificar modalidades concretas de racismo, respecto de las cuales desarrollar prácticas específicas de intervención en ámbitos, o niveles, particulares dentro del relativamente amplio campo de la Educación Superior. El alcance y características de esas prácticas orientadas a la erradicación del racismo, podrían resultar apropiadas para lograr resultados en los contextos concretos de actuación de los diversos tipos de actores sociales, sus posiciones en ellos y sus posibilidades concretas de acción.

En concreto, la Declaración de la CRES 2018 incluye recomendaciones sobre el tema que nos ocupa tanto respecto de las políticas, como de las instituciones de 
Educación Superior. La capacidad de incidir en el diseño de políticas de Educación Superior que tomen en cuenta esas recomendaciones reside en buena medida en los gobiernos y sus agencias, y dependiendo de las dinámicas políticas propias de cada sociedad también de las autoridades de las IES. Otro tanto cabe decir de las asignaciones presupuestarias que las pongan en práctica. Adicionalmente, y nuevamente dependiendo de dinámicas políticas, las acciones de unos y otras pueden resultar más o menos permeables a demandas y propuestas de organizaciones de docentes y estudiantes, así como de diversos sectores y organizaciones sociales, incluyéndolas de pueblos indígenas y afrodescendientes. En cambio, la puesta en práctica de las recomendaciones de la Declaración directamente orientadas a las IES depende principalmente del accionar de los actores propios de estas, es decir, de manera simplificada, de sus autoridades, docentes, estudiantes, y trabajadores no-docentes. Según los casos, en la puesta en práctica de esas recomendaciones también podrían tener incidencia las organizaciones de egresados, los colegios profesionales, y diversos sectores empresariales y organizaciones sociales, incluyendo las de los pueblos en cuestión.

Para erradicar el racismo de la Educación Superior, operativamente resulta útil comprender que existen problemas de "racismo estructural" que no son específicos de su ámbito sistémico, ni de las IES. Estos factores no pueden ser resueltos en/desde este, o al menos no solo en/desde este campo e instituciones, si bien no por ello pueden obviar su importancia. Por el contrario, es necesario buscar formas de contribuir a responder a ellos.

Ejemplos de este tipo de factores son las desventajas históricamente acumuladas que provienen del despojo de los territorios de los pueblos indígenas iniciado durante la invasión y colonización europea, y continuado en las repúblicas poscoloniales. Esto les obligó a desplazarse, y en algunos casos fueron desplazados de manera forzada por fuerzas militares. Como consecuencia de ello se vieron privados de sus fuentes de alimentos y cobijo, y se vieron obligados a buscar nuevas formas de sustento. Problemas semejantes han afectado a las comunidades afrodescendientes que tras huir de la esclavitud, o culminada esta, establecieron espacios territoriales de subsistencia (quilombos, cumbés, o palenques, entre otras denominaciones, en diversos países) de los que en muchos casos posteriormente 
fueron expulsados. Con variantes de forma, estos tipos de problemas continúan afectando a comunidades de pueblos indígenas y afrodescendientes (ONU, , 2005, 2010, 2013, 2014a, 2015).

Estos procesos complejos y de larga evolución histórica tienen diversos tipos de consecuencias, pero en lo referente al campo educativo se expresan, por ejemplo, en que las tasas de analfabetismo, como también las de educación primaria o secundaria incompleta, registran valores más elevados entre la población indígena y afrodescendiente, que los correspondientes a otros sectores de población de cada uno de los países de la región. Estos problemas generan inequidad y exclusión, y constituyen causas estructurales de la escasa participación de personas indígenas y afrodescendientes entre el estudiantado, cuerpos docentes, autoridades y funcionarios de las universidades y otras IES. Es relativamente poco lo que se puede hacer de manera directa e inmediata desde las universidades y otras IES para lograr revertir estos problemas. En cambio, se puede contribuir a hacerlo desde una perspectiva de más largo aliento.

Algunas universidades y otras IES de varios países de la región ya hace tiempo que desarrollan valiosas iniciativas orientadas a responder a estos problemas (MATO, 2008, 2017, 2018, 2019). Pero suelen hacerlo con escasos recursos, sin exigir de manera categórica a los gobiernos los presupuestos necesarios para sostenerlas. En este sentido parece existir una omisión grave. Tanto los gobiernos como numerosas universidades y otras IES parecen olvidar que asegurar oportunidades educativas a personas y comunidades de estos pueblos, a todos los niveles, en condiciones equitativas, y que resulten apropiadas a sus contextos e intereses, constituyen derechos establecidos en los instrumentos internacionales comentados anteriormente en este artículo y en las constituciones nacionales y leyes de la mayoría de los países de la región. Avanzar en esta dirección puede resultar más viable si, además de enfocar en los problemas de racismo estructural, se procura resolver los de carácter sistémico e institucional.

Existen problemas de racismo que cabe caracterizar como de carácter sistémico, en tanto son propios de las normas, políticas y prácticas de cada sistema de educación superior en particular. Los factores sistémicos que aseguran la reproducción del racismo generalmente no resultan evidentes. Por ejemplo, las 
normativas vigentes establecen la necesidad de contar con título universitario o de Educación Superior para dar clase en las instituciones de ese nivel, lo cual en principio puede considerarse como un requisito "normal". El problema es que este tipo de normas, por ejemplo, impide que los mejores hablantes de las lenguas de pueblos indígenas las enseñen en el ámbito de la Educación Superior. Esta limitación menoscaba las posibilidades de formar más y mejores docentes para los sistemas de educación intercultural bilingüe, y esto a su vez afecta las posibilidades de que niñas, niños y jóvenes de comunidades de estos pueblos reciban educación apropiada y de calidad. Limitaciones semejantes impiden que sabias/os de las comunidades enseñen en carreras de salud sus conocimientos acerca de aplicaciones terapéuticas de ciertas especies vegetales. Problemas semejantes se presentan en diversas carreras respecto de conocimientos de manejo ambiental, sistemas de justicia, y resolución de conflictos, entre otros.

Finalmente, existen otros tipos de factores propios de los diseños y prácticas institucionales de cada universidad o IES en particular Ejemplos de estos tipos de factores que cabe caracterizar como de racismo institucional son aquellos asociados a la oferta de carreras de cada institución, sus planes de estudio, y las actividades de aprendizaje institucionalmente acreditables, entre otros. Son escasas las universidades y otras IES que diseñan y ofrecen carreras y planes de estudio que resulten pertinentes con la diversidad cultural de los territorios en que están establecidas, y aún menos las que ofrecen oportunidades de formación relevantes para las necesidades y demandas de comunidades de pueblos indígenas y afrodescendientes. Esto resulta especialmente preocupante en los casos de algunas carreras como por ejemplo las de salud, en las que solo muy excepcionalmente se ofrece formación intercultural. En América Latina, donde aproximadamente 8\% de la población se autoreconoce como miembro de algún pueblo indígena y casi $22 \%$ lo hace como afrodescendiente, la mayoría de las y los profesionales de Medicina y Enfermería se gradúan sin tener formación sobre las representaciones de salud y enfermedad de pueblos indígenas y afrodescendientes, y frecuentemente tampoco sobre enfermedades que son endémicas de estas poblaciones. 


\section{La erradicación del racismo en Educación Superior demanda trabajar sobre factores estructurales, sistémicos, institucionales y subjetivos}

Erradicar el racismo de la Educación Superior resulta crucial para lograr hacerlo en todos los ámbitos sociales, porque los egresados de este nivel educativo alcanzan posiciones clave en todas las sociedades contemporáneas, tanto como dirigentes políticos, como sociales y empresariales. Además pasan a ocupar posiciones clave en los sistemas educativos, de salud y de justicia. De especial importancia resulta el papel de docentes y comunicadores sociales, en tanto formadores de ciudadanos y de opinión pública.

Para erradicar el racismo de la Educación Superior es necesario desplegar acciones específicas encaminadas a combatirlo en todas sus modalidades y manifestaciones. La idea de "racismo estructural" puede no resultar suficientemente eficaz a los fines operativos. Tampoco es posible reducir los problemas del racismo a las manifestaciones explícitas de "discriminación racial” Es necesario identificar las modalidades de racismo sistémicas e institucionales, hacerlas visibles y promover la reflexión y el debate sobre ellas. Para combatir el racismo resulta útil aprender de la experiencia de la lucha contra el machismo.

Ambas ideologías impregnan la vida de todas las sociedades contemporáneas. Anidan y producen efectos en todos los ámbitos sociales, también en la Educación Superior. Las formas en que estas ideologías se manifiestan no son solo las de discriminación y violencia, pero estas también existen. Promover en las IES y sistemas de Educación Superior la adopción de protocolos de prevención y sanción de la discriminación y violencia étnico/racial puede resultar una vía para visibilizar estos problemas, para promover reflexiones y debates. Esto puede servir para revisar los planes de estudio, los programas de las asignaturas y sus bibliografías, así como las líneas de investigación que reciben mayor atención y financiamiento. También es necesario avanzar en la producción de estadísticas que den visibilidad a estos grupos de población. Ya se ha dado este paso en los censos nacionales de casi 
todos los países de la región, resulta necesario darlo en las IES y sistemas de Educación Superior.

\section{Referencias}

CEPAL - Comisión Económica para América Latina y el Caribe. La matriz de la desigualdad social en América Latina. Santiago: CEPAL - Comisión Económica para América Latina y el Caribe, 2016.

CRES - III Conferencia Regional de Educación Superior de América Latina y el Caribe. Declaración. Córdoba, Argentina, 14 de junio de 2018. Disponible en: http://espacioenlaces.org/wpcontent/uploads/2018/07/declaracion_cres2018.pdf.

Acceso en :02/11/2019

BELLO, A.; RANGEL, M. Etnicidad, "raza" y equidad en América Latina y el Caribe. Santiago: CEPAL, 2000.

HOPENHAYN, M.; BELLO, A.; MIRANDA, F. Los pueblos indígenas y afrodescendientes ante el nuevo milenio. Santiago: CEPAL, 2006.

MATO, D. (org). Diversidad cultural e interculturalidad en Educación Superior: Experiencias en América Latina. Caracas: Instituto Internacional de la UNESCO para la Educación Superior en América Latina y el Caribe (UNESCO - IESALC), 2008.

MATO, D. (org.). Educación Superior y Pueblos Indígenas y Afrodescendientes en América Latina: Normas, Políticas y Prácticas. Caracas: UNESCO - IESALC, 2012.

MATO, D. (org.) Educación Superior y Pueblos Indígenas en América Latina: Políticas y prácticas de inclusión, democratización e interculturización. Sáenz Peña (Pcia. de Buenos Aires): EDUNTREF; José María Morelos, Q. R., México: UIMQRoo, 2017.

MATO, D. (org.). Educación Superior, diversidad cultural e interculturalidad en América Latina. Caracas: UNESCO - IESALC y Córdoba: Universidad Nacional de Córdoba, 2018.

MATO, D. (org.). Educación Superior y Pueblos Indigenas en América Latina - Colaboración intercultural: experiencias y aprendizajes. Sáenz Peña (Pcia. de Buenos Aires): EDUNTREF, 2019.

OIT - Organización Internacional del Trabajo. Convenio 169 de la sobre Pueblos Indígenas y Tribales en Países Independientes, 1989. Disponible en: https://www.ilo.org/dyn/normlex/es/f?p=NORMLEXPUB:12100:0::NO::P12100_ILO_CODE: C169. Acceso en: 02/11/2019

ONU - Organización de Naciones Unidas. Declaración Universal de Derechos Humanos, 1948. Disponible en: https://www.un.org/es/documents/udhr/UDHR_booklet_SP_web.pdf. Acceso en:02/11/2019

ONU - Convención Internacional sobre la Eliminación de todas las Formas de Discriminación Racial, $1965 . \quad$ Disponible en: https://www.ohchr.org/SP/ProfessionalInterest/Pages/CERD.aspx. Acceso en:02/11/2019 
ONU - Pacto Internacional de Derechos Económicos, Sociales y Culturales, 1966. Disponible en: https://www.ohchr.org/sp/professionalinterest/pages/cescr.aspx. Acceso en: $02 / 11 / 2019$

ONU - Declaración de Naciones Unidas sobre los derechos de las personas pertenecientes a minorías nacionales o étnicas, religiosas y lingüísticas, 1992. Disponible en: https://www.ohchr.org/sp/professionalinterest/pages/minorities.aspx. Acceso en: $02 / 11 / 2019$

ONU - Report of the Special Rapporteur on the situation of human rights and fundamental freedoms of indigenous people, Rodolfo Stavenhagen (E/CN.4/2005/88), 2005. Disponible en: https://documents-dds-ny.un.org/doc/UNDOC/ GEN/G04/169/40/PDF/G0416940.pd??OpenElement. Acceso en:02/11/2019

ONU - Report of the Working Group of experts on people of African Descent. Visit to Ecuador (A/HRC/13/59), 2010. Disponible en: https://documents-ddsny.un.org/doc/UNDOC/GEN/G10/102/07/PDF/G1010207.pdf?OpenElement.

Acceso en:02/11/2019

ONU - Report of the Working Group of Experts on People of African Descent on its twelfth session. Mission to Panama (A/HRC/24/52/Add.2), 2013. Disponible en: https://documents-dds-ny.un.org/doc/UNDOC/GEN/G13/164/35/PDF/G1316435.pdf?OpenElement. Acceso en:02/11/2019

ONU - Report of the Working Group of Experts on People of African Descent on its fourteenth session. Mission to Brazil (A/HRC/27/68/Add), 2014a. Disponible en: https://documents-dds ny.un.org/doc/UNDOC/GEN/G14/168/64/PDF/G1416864.pdfPOpenElement Acceso en:02/11/2019

ONU Programa de actividades del Decenio Internacional para los Afrodescendientes (A/RES/69/16), 2014b. Disponible en: https://undocs.org/es/A/RES/69/16. Acceso en $: 02 / 11 / 2019$

ONU - Report of the Working Group of Experts on People of African Descent. Visit to Argentina (A/HRC/42/59/Add.2), 2019. Disponible en: https://documents-ddsny.un.org/doc/UNDOC/GEN/G19/242/57/PDF/G1924257.pdf?OpenElement.

Acceso en:02/11/2019

REDING, S. Diversidad y racismo en América Latina Latinoamérica. Revista de Estudios Latinoamericanos, v. 44, n. 1, p. 157-179, 2007.

RECIBIDO: 08/04/2020 APROBADO: $11 / 05 / 2020$

RECEIVED: 04/08/2020

APPROVED: 05/11/2020

RECEBIDO: 08/04/2020

APROVADO: $11 / 05 / 2020$ 